\title{
Promoter methylation of ADAMTS1 and $B N C 1$ as potential biomarkers for early detection of pancreatic cancer in blood
}

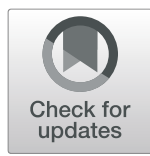

\author{
Maryam A. L. Eissa ${ }^{1 \dagger}$, Lane Lerner ${ }^{1 \dagger}$, Eihab Abdelfatah ${ }^{1}$, Nakul Shankarr ${ }^{1}$, Joseph K. Canner ${ }^{1}$, Nesrin M. Hasan ${ }^{7}$, \\ Vesal Yaghoobi ${ }^{1}$, Barry Huang ${ }^{1}$, Zachary Kerner ${ }^{1}$, Felipe Takaesu', Christopher Wolfgang ${ }^{1}$, Ruby Kwak', \\ Michael Ruiz', Matthew Tam¹, Thomas R. Pisanic II3, Christine A. lacobuzio-Donahue ${ }^{4,5}$, Ralph H. Hruban²,5, \\ Jin He ${ }^{1}$, Tza-Huei Wang ${ }^{3}$, Laura D. Wood ${ }^{2,5,6}$, Anup Sharma ${ }^{1,7}$ and Nita Ahuja ${ }^{1,2,6,7^{*}}$
}

\begin{abstract}
Background: Despite improvements in cancer management, most pancreatic cancers are still diagnosed at an advanced stage. We have recently identified promoter DNA methylation of the genes ADAMTS1 and BNC1 as potential blood biomarkers of pancreas cancer. In this study, we validate this biomarker panel in peripheral cell-free tumor DNA of patients with pancreatic cancer.

Results: Sensitivity and specificity for each gene are as follows: ADAMTS1 87.2\% and 95.8\% (AUC=0.91;95\% Cl 0 . 71-0.86) and BNC1 64.1\% and 93.7\% (AUC = 0.79; 95\% Cl 0.63-0.78). When using methylation of either gene as a combination panel, sensitivity increases to $97.3 \%$ and specificity to $91.6 \%$ (AUC $=0.95 ; 95 \% \mathrm{Cl} 0.77-0.90$ ). Adding pre-operative CA 19-9 values to the combined two-gene methylation panel did not improve sensitivity. Methylation of ADAMTS1 was found to be positive in $87.5 \%$ (7/8) of stage I, $77.8 \%$ (7/9) of stage IIA, and 90\% (18/20) of stage IIB disease. Similarly, BNC1 was positive in $62.5 \%$ (5/8) of stage I patients, $55.6 \%(5 / 9)$ of stage IIA, and $65 \%(13 / 20)$ of patients with stage IIB disease. The two-gene panel (ADAMTS1 and/or BNC1) was positive in 100\% (8/8) of stage I, $88.9 \%(8 / 9)$ of stage IIA, and $100 \%$ (20/20) of stage IIB disease. The sensitivity and specificity of the two-gene panel for localized pancreatic cancer (stages I and II), where the cancer is eligible for surgical resection with curative potential, was $94.8 \%$ and $91.6 \%$ respectively. Additionally, the two-gene panel exhibited an AUC of 0.95 (95\% Cl 0.90-0.98) compared to $57.1 \%$ for CA $19-9$ alone.

Conclusion: The methylation status of ADAMTS1 and BNC1 in cfDNA shows promise for detecting pancreatic cancer during the early stages when curative resection of the tumor is still possible. This minimally invasive blood-based biomarker panel could be used as a promising tool for diagnosis and screening in a select subset of high-risk populations.
\end{abstract}

Keywords: Cell-free DNA, MOB, ADAMTS1, BNC1, Methylation, Biomarker, Pancreatic cancer

\footnotetext{
*Correspondence: nita.ahuja@yale.edu

Maryam AL Eissa and Lane Lerner contributed equally.

${ }^{1}$ Department of Surgery, The Johns Hopkins University School of Medicine,

Baltimore, MD, USA

${ }^{2}$ Department of Oncology, The Sidney Kimmel Comprehensive Cancer

Center, The Johns Hopkins University School of Medicine, Baltimore, MD,

USA

Full list of author information is available at the end of the article
}

(c) The Author(s). 2019 Open Access This article is distributed under the terms of the Creative Commons Attribution 4.0 International License (http://creativecommons.org/licenses/by/4.0/), which permits unrestricted use, distribution, and reproduction in any medium, provided you give appropriate credit to the original author(s) and the source, provide a link to the Creative Commons license, and indicate if changes were made. The Creative Commons Public Domain Dedication waiver (http://creativecommons.org/publicdomain/zero/1.0/) applies to the data made available in this article, unless otherwise stated. 


\section{Introduction}

Pancreatic cancer is the third leading cause of both domestic and global cancer-related mortality [1]. It has a poor prognosis with an overall 5-year survival rate of $7 \%$ $[2,3]$, as the cancer often grows insidiously and initially does not cause symptoms. The poor prognosis is at least in part due to the absence of specific symptoms early in the course of the disease and lack of effective diagnostic methods. Over $75 \%$ of pancreatic cancer cases are diagnosed at stage III/IV [4]. Yet, surgical resection is currently the only potentially curable therapy $[5,6]$. Therefore, there is an urgent need for reliable, non-invasive, and cost-effective early detection method for biomarkers of pancreatic cancer.

DNA methylation plays an important role in cancer development and progression [7]. DNA methylation can alter the DNA chromatin structure and can lead to the silencing of tumor suppressor genes or activation of oncogenes [7, 8]. Many of these changes occur early in tumorigenesis, making epigenetic modifications a promising target as biomarkers for the early detection of cancer [9-12]. DNA methylation changes occur early in cancer formation for many cancer types including colorectal [13], breast [14], and pancreas [10-12, 15] and that methylation changes can be potent biomarkers for early detection [16-18]. The recent FDA approval of methylation biomarkers for colorectal cancer (Cologuard $^{\circ}$, Epi proColon ${ }^{\circ}$ ) as well as bladder cancer (Exact Sciences Inc.) serves as a blueprint for taking these biomarkers to patient care. In pancreatic cancer, CA19-9, a carcinoembryonic antigen, is approved by the FDA for prognostic surveillance of known pancreatic cancer patients; however, it is considered to have low sensitivity and specificity for pancreatic cancer detection [19-22]. Currently approved tests for the early detection of pancreatic cancer do not exist.

Cell-free DNA (cfDNA) from tumor tissues has been found within plasma samples from cancer patients' peripheral circulating blood. Additionally, the amount of total cfDNA in cancer patients is higher than in normal populations [23-27]. This cfDNA is composed of short segments of nucleic acids that are not associated with cells or cell fragments. Importantly, cfDNA reflects the genetic and epigenetic makeup of the tumor from which it originates, making it a desirable and highly specific biomarker for the early detection of cancer. Tests for circulating tumor DNA can be improved when they are combined with highly selected protein markers [26].

We have been investigating the role of epigenetic changes in pancreatic cancer over the past few years and have previously published on the widespread methylation changes that occur in pancreatic cancer. Our studies identified two biomarkers ADAMTS1 (A disintegrin and metalloproteinase with thrombospondin motifs 1) and BNC1 (zinc finger protein basonuclin-1) as highly sensitive markers for the early detection of pancreatic cancer in tissue [15]. In a previous study of selected pancreatic cancer patients, we reported promising sensitivity and specificity with the two genes at $81 \%$ and $85 \%$ respectively using non-invasive fluids [15].

In this study, we took advantage of a newly developed $[28,29]$ highly reliable technique called methylation on beads (MOB). DNA methylation of genes ADAMTS1 and $B N C 1$ was studied using cfDNA in a large cohort of patients with varying stages of pancreatic cancer and an age-matched normal group to determine the sensitivity, specificity, and applicability of this two-gene panel as a non-invasive biomarker set for the early detection of pancreatic cancer. Furthermore, pre-operative CA 19-9 levels were also compiled to study the significance of their values, either alone or in combination with the methylation-based gene panel, in detecting early stage pancreatic cancer.

\section{Results}

\section{Patient and control cohorts}

Patients with different stages of pancreatic cancer undergoing surgical resection were enrolled in this study. $20.5 \%(8 / 39)$ were found to have stage I cancer, $23.1 \%$ (9/39) stage IIA, $51.3 \%(20 / 39)$ stage IIB, and only $5.1 \%$ $(2 / 39)$ stage III/IV cancers. Patients with stage III/IV cancers were surgically explored and the surgery aborted due to the advanced stage of the disease. There was no significant age difference between the cancer and control groups (mean age 60.1 years vs. 65.5 years) (Table 1). Cigarette smoking, pancreatitis, and diabetes mellitus have all been identified as important risk factors for pancreatic cancer in previous studies [30-32]. Our study recruited population-based matched controls to determine the specificity of our markers. Of note, amongst the controls, $48 \%$ had diabetes mellitus, 38\% had hypertension, and $17 \%$ were current smokers. Patients with pancreatitis were undergoing surgery for pain control that was unresponsive to long-standing medical therapy. Patients with pancreatitis had median 84 months of disease prior to undergoing surgery. There was a significant racial difference between the two cohorts used in the study $(76 \%$ of control group were African-American vs $5 \%$ of cancer cases; $p<0.05)$ (Table 1 ). The majority of the cancer patients $(69.2 \%)$ were treated with pancreaticoduodenectomy, while $10.3 \%$ and $15.4 \%$ had total and distal pancreatectomy respectively, and in $5.1 \%$, the resection was aborted (Additional file 1: Table S1).

\section{ADAMTS1 and BNC1 methylation status in pancreatic cancer} cfDNA isolated from the blood samples obtained from pancreatic cancer patients $(n=39)$, pancreatitis $(n=8)$, and control group $(n=95)$ was evaluated (Fig. 1). Overall methylation of either gene (ADAMTS1 and/or BNC1) 
Table 1 Patient demographics, tumor clinicopathologic features

\begin{tabular}{|c|c|c|c|c|}
\hline & Control $(n=95)$ & Cancer $(n=39)$ & $p$ value (cancer vs control) & Pancreatitis $(n=8)$ \\
\hline Average age, years (range) & 65.5 (21-96) & $60.1(29-83)$ & 0.059 & $46.6(29-70)$ \\
\hline \multicolumn{5}{|l|}{ Gender } \\
\hline Male (\%) & $41(43 \%)$ & $26(67 \%)$ & 0.013 & $4(50 \%)$ \\
\hline Female (\%) & $54(57 \%)$ & $13(33 \%)$ & & $4(50 \%)$ \\
\hline \multicolumn{5}{|l|}{ Race } \\
\hline White (\%) & $22(23 \%)$ & $34(87 \%)$ & & $6(78 \%)$ \\
\hline Black (\%) & $72(76 \%)$ & $2(5 \%)$ & $<0.05$ & $1(11 \%)$ \\
\hline Others (\%) & $0(0 \%)$ & $3(8 \%)$ & & $1(11 \%)$ \\
\hline Not specified (\%) & $1(1 \%)$ & $0(0 \%)$ & & $0(0 \%)$ \\
\hline CA 19-9 level* & - & $n=34$ & N/A & $n=1$ \\
\hline Average & - & 665.3 & & - \\
\hline Median & - & 47.6 & & - \\
\hline Highest & - & $12,302.5$ & & - \\
\hline Lowest & - & 1 & & 16.1 \\
\hline Smoking status & $n=95$ & $n=39$ & & $n=8$ \\
\hline Never & $38(40 \%)$ & $22(56 \%)$ & & $5(63 \%)$ \\
\hline Former & $40(42 \%)$ & $10(26 \%)$ & 0.159 & $3(38 \%)$ \\
\hline Current & $16(17 \%)$ & $7(18 \%)$ & & $0(0 \%)$ \\
\hline Not specified & $1(1 \%)$ & $0(0 \%)$ & & $0(0 \%)$ \\
\hline Average tumor size $(\text { range, } \mathrm{cm})^{* *}$ & - & $3.2(0.9-8)$ & N/A & - \\
\hline Differentiation & & $n=39$ & $\mathrm{~N} / \mathrm{A}$ & \\
\hline Well & - & $1(3 \%)$ & & - \\
\hline Moderate & - & $17(44 \%)$ & & - \\
\hline Poor & - & $12(31 \%)$ & & - \\
\hline Undifferentiated & - & $2(5 \%)$ & & - \\
\hline Not specified & - & $7(18 \%)$ & & - \\
\hline
\end{tabular}

${ }^{*}$ CA 19-9: five values unavailable for PDAC patient and four unavailable for pancreatitis

**Tumor size: three PDAC, no resection done, N/A: Not applicable



was observed in $97.4 \%$ of cancer patients (38/39) compared to $8.4 \%$ of control patients (8/95). Methylation of ADAMTS1 was detected in $87.2 \%$ (34/39) pancreatic adenocarcinomas. BNC1 methylation was detected in $65.1 \%(25 / 39)$ of cases. Methylation analysis was also performed in a series of non-cancer individuals $(n=95)$. Amongst this group, methylation of ADAMTS1 was detected in $4.2 \%(4 / 95)$, while $B N C 1$ was detected in $6.3 \%$ (6/95) of cases. 97.4\% (38/39) and 8.4\% (8/95) of patients and controls respectively showed evidence of methylation of either of the two genes. Among controls, 17\% and $42 \%$ of patients were current and former smokers respectively, while $55.4 \%$ were type II diabetic. The use of the two-gene combination (ADAMTS1 and/or BNC1) panel made a demonstrable difference of the predictive power of both the genes as compared to the use of a single gene by itself (Table 2). Using the presence of methylation in either gene, pancreatic cancer was detected in 97.4\% (38/39) of all pancreatic cancer patients. 
Table 2 Methylation frequency of two-gene panel (ADAMTS1 and/or BNC1) vs frequency of CA 19-9 elevation in control, PDAC, and pancreatitis

\begin{tabular}{|c|c|c|c|c|c|c|}
\hline \multirow[t]{2}{*}{ Patients $(n)$} & \multicolumn{2}{|l|}{ Control } & \multicolumn{2}{|l|}{ PDAC } & \multicolumn{2}{|l|}{ Pancreatitis } \\
\hline & Positive/total & Positive (\%) & Positive/total & Positive (\%) & Positive/total & Positive (\%) \\
\hline ADAMTS1 & $4 / 95$ & 4.2 & $34 / 39$ & 87.2 & $6 / 8$ & 75 \\
\hline$B N C 1$ & $6 / 95$ & 6.3 & $25 / 39$ & 65.1 & $4 / 8$ & 50 \\
\hline ADAMTS1 and BNC1 & 2/95 & 2.1 & $21 / 39$ & 53.8 & $3 / 8$ & 37.5 \\
\hline ADAMTS1 and/or BNC1 & $8 / 95$ & 8.0 & $38 / 39$ & 97.4 & $7 / 8$ & 87.5 \\
\hline CA $19-9(n=34)$ & \multicolumn{2}{|c|}{ N/A } & $19 / 34$ & 55.9 & \multicolumn{2}{|c|}{ N/A } \\
\hline ADAMTS1 and BNC1 and CA 19-9* & \multicolumn{2}{|c|}{ N/A } & $6 / 34$ & 17.6 & \multicolumn{2}{|c|}{ N/A } \\
\hline ADAMTS1 and/or BNC1 + CA 19-9 & \multicolumn{2}{|c|}{ N/A } & $38 / 39$ & 97.4 & \multicolumn{2}{|c|}{ N/A } \\
\hline
\end{tabular}

*Positive for all three, N/A: Not applicable

We also investigated the utility of CA19-9 as an early detection biomarker. CA19-9 values were available in 87.2\% (34/39) pancreatic cancer patients. Amongst these patients, CA19-9 was elevated above the normal threshold (> 36 units) in $55.9 \%$ (19/34) of pancreatic adenocarcinoma group. Thus, CA19-9 failed to detect the presence of pancreatic cancer in $44.1 \%(15 / 34)$ of the cancer cases. We next tested the utility of CA19-9 as an additive biomarker. The combined sensitivity of ADAMTS1 and CA $19-9$ was $87.2 \%$ compared to $87.2 \%$ for ADAMTS1 alone while that of $B N C 1$ with CA $19-9$ was $89.2 \%$ compared to $64.1 \%$ with $B N C 1$ alone. However, there was no improvement in sensitivity on the addition of CA 19-9 to the two-gene methylation panel (ADAMTS1 and/or BNC1 +/- CA19-9, 97.4\% both) (Table 2).

\section{ADAMTS1 and BNC1 methylation status in various stages of cancer}

Methylation of ADAMTS1 was found to be positive in cfDNA of $87.5 \%$ (7/8) patients with stage I cancer, $77.8 \%$ (7/9) of stage IIA, $90 \%(18 / 20)$ of stage IIB, and $100 \%$ $(2 / 2)$ of stage III/IV pancreatic cancer patients. Similarly, $B N C 1$ was positive in $62.5 \%(5 / 8)$ of stage I patients, $55.6 \%(5 / 9)$ of stage IIA, $65 \%(13 / 20)$ of stage IIB, and $100 \%(2 / 2)$ of stage III/IV pancreatic cancers. When considering the combination panel of both genes (ADAMTS1 and/or BNC1), the combined panel was positive in $100 \%(8 / 8)$ of stage I, $88.9 \%$ (8/9) of stage
IIA, $100 \%(20 / 20)$ of stage IIB, and $100 \%(2 / 2)$ of stages III and IV pancreatic cancers (Table 3 ). The difference in positive methylation prevalence between different stages was not statistically significant. When looking at CA19-9 by different cancer stages, CA19-9 was positive in only $57.1 \%(4 / 7)$ of stage I patients, $44.4 \%(4 / 9)$ of stage IIA, $64.7 \%(11 / 17)$ of stage IIB, and $0 \%(2 / 2)$ of stage III/IV pancreatic cancers. The combined two-gene panel of ADAMTS1 and/or BNC1 detected 94.8\% (37/39) of all early-stage PDAC patients (stages I and II) who are eligible for surgical curative resection vs $57.6 \%$ for CA19-9 $(19 / 33)$ alone $(p=0.0003)$.

\section{Evaluation of the two-gene panel in pancreatitis}

Patients with pancreatitis have a higher risk of developing PDAC; therefore, cfDNA samples from eight patients with chronic pancreatitis were also evaluated. Using the combination panel (ADAMTS1 and/or BNC1), 87.5\% $(7 / 8)$ of chronic pancreatitis patients had positive methylation (Table 2).

\section{Sensitivity, specificity, and the accuracy of the biomarker} We calculated a methylation positivity for each gene independently and as a combination panel. There was no significant difference in methylation level between different races, genders, or age groups. We then used these values to generate a receiver operating characteristic curve (ROC) to determine the sensitivity and specificity

Table 3 Methylation frequency of two-gene panel (ADAMTS1 and/or BNC1) vs frequency of CA 19-9 elevation in different stages of pancreatic ductal adenocarcinoma

\begin{tabular}{|c|c|c|c|c|c|c|c|c|}
\hline \multirow[t]{2}{*}{ Patients } & \multicolumn{2}{|l|}{ Stage I (\%) } & \multicolumn{2}{|l|}{ Stage IIA (\%) } & \multicolumn{2}{|l|}{ Stage IIB (\%) } & \multicolumn{2}{|c|}{ Stages III-IV (\%) } \\
\hline & Positive/total & Positive (\%) & Positive/total & Positive (\%) & Positive/total & Positive (\%) & Positive/total & Positive (\%) \\
\hline ADAMTS1 & $7 / 8$ & 87.5 & $7 / 9$ & 77.8 & $18 / 20$ & 90 & $2 / 2$ & 100 \\
\hline$B N C 1$ & $5 / 8$ & 62.5 & $5 / 9$ & 55.6 & $13 / 20$ & 65 & $2 / 2$ & 100 \\
\hline ADAMTS1 and/or BNC1 & $8 / 8$ & 100 & $8 / 9$ & 88.9 & $20 / 20$ & 100 & $2 / 2$ & 100 \\
\hline CA $19-9(n=34)^{*}$ & $4 / 7$ & 57.1 & $4 / 9$ & 44.4 & $11 / 17$ & 64.7 & $0 / 1$ & 0 \\
\hline ADAMTS1 and/or BNC1 + CA 19-9 & $8 / 8$ & 100 & $8 / 9$ & 88.9 & $20 / 20$ & 100 & $2 / 2$ & 100 \\
\hline
\end{tabular}

*Missing two CA 19-9 values, so \% out of total recorded CA 19-9 values for each stage 
for each gene at the optimal cutoff value. The sensitivity and specificity of ADAMTS1 was $87.2 \%$ and $95.8 \%$, respectively. $B N C 1$ demonstrated a sensitivity and specificity of $64.1 \%$ and $93.7 \%$, respectively. The combination panel of methylation of either gene demonstrated a sensitivity and specificity of $97.4 \%$ and $91.6 \%$, respectively. The area under the curve (AUC) for ADAMTS1 was 0.91 (95\% CI 0.85-0.95), while AUC of BNC1 was 0.79 (95\% CI 0.70-0.85). The AUC of the two-gene combination panel was 0.95 (95\% CI 0.91-0.98) (Table 4) (Fig. 2a-c). The AUC for early stage, resectable pancreatic cancers was 0.91 (95\% CI 0.85-0.95) for ADAMTS1, AUC 0.78 (95\% CI 0.70-0.85) for BNC1 alone, while the AUC of the two-gene combination was 0.95 (95\% CI 0.90-0.98).

\section{Discussion}

DNA methylation plays an important role in the epigenetic modification of the cells. Hypermethylation of CpG islands in promoter regions of tumor suppressor genes can lead to gene downregulation, gene silencing, or aberrant post-translational modifications, all of which can contribute to cancer development. Identification of reliable early detection markers to identify cancers in a non-invasive fashion using DNA methylation markers has shown exciting promise.

An array of noninvasive clinical tests has been developed for colorectal (CRC) cancer screening, including the fecal occult blood test, the fecal immunochemical test, the fecal-based DNA test, and the blood-based DNA test (the SEPT9 assay). Another popular commercially available test is a non-invasive colon cancer screening test based on DNA obtained from the stool, Cologuard $^{\circ}$ (Exact Sciences). This test takes a multi-target approach and screens for altered DNA, hemoglobin biomarkers, methylation of genes (NDRG4 and $B M P 3$ ), and KRAS point mutations for early detection of CRC [33]. Epi proColon ${ }^{\circ}$ based on methylated septin 9 which is altered in colorectal cancer tumor cells more often than in normal cells is the first and only FDA-approved blood-based test the detection of colorectal cancer [34-36]. Another commercial test, Oncotype DX, based on a multigene approach, has been developed for the early detection of breast [37], colon [38], and prostate [39] cancers. However, given the fact that one gene rarely defines the status of tumor formation, a multi-gene approach has been used in order to develop a detection method that can recognize early events in tumorigenesis [40, 41].

Early detection of cancer is crucial to treatment. Early detection can mean less invasive or risky surgical procedures prior to metastasis, along with improved survival. This is exemplified by a diversity of public health statistics on cancer. For breast cancer, the 5-year survival of women with early stage diagnosis is as high as $90 \%$, but as low as $15 \%$ with advanced disease [42]. Early detection through screening has increased the 5-year survival rate to $88 \%$ and $90 \%$ in lung and CRC respectively [43, 44]. Early detection of pancreatic cancer is also associated with better prognosis [45-47].

Earlier detection holds great promise for patients with pancreatic cancer [48]. Chemotherapy has only been shown to have moderate success, and surgical resection remains the only curative option but less than $20 \%$ of patients present with resectable tumor $[46,49,50]$. Additionally, the most important factor in determining whether a patient is a candidate for surgical intervention is the presence or absence of metastases. However, pancreatic cancer is often either discovered incidentally or remains asymptomatic for a long period, growing to become either locally advanced or metastatic before causing symptoms such as gastric pain, jaundice, or other signs of biliary obstruction. At that point, $80-85 \%$ of patients present with already advanced, unresectable disease. Developing an early diagnostic approach is one possible solution to increase detection at resectable stages of disease [25-27]. Most studies concerning the use of tumor markers in pancreatic cancer have been directed toward the use of serum-based testing. However, currently, no available serum-based marker can be used to aid in the diagnosis of pancreatic cancer because of suboptimal sensitivity and specificity. There is a dire need for an early detection test that exhibits a high sensitivity and specificity. To achieve this, the focus of early detection biomarkers should be to identify early stages (stages I and II) of the disease.

Table 4 Gene methylation detection in plasma samples for cancer and controls

\begin{tabular}{|c|c|c|c|c|c|}
\hline & Sensitivity & Specificity & PPV & NPV & $A \cup C$ \\
\hline CA19-9 & $55.9 \%(19 / 34)$ & NA & NA & NA & NA \\
\hline ADAMTS1 & $87.2 \%(34 / 39)$ & $95.8 \%(91 / 95)$ & $89 / 5 \%(34 / 38)$ & $95.8 \%(91 / 96)$ & 0.91 \\
\hline$B N C 1$ & $64.1 \%(25 / 39)$ & $93.7 \%(89 / 95)$ & $80.6 \%(25 / 31)$ & $86.4 \%(89 / 103)$ & 0.79 \\
\hline ADAMTS1 and/or BNC1 & $97.4 \%(38 / 39)$ & $91.6 \%(87 / 95)$ & $82.6 \%(38 / 46)$ & $98.9 \%(87 / 88)$ & 0.95 \\
\hline ADAMTS1 and/or BNC1 and/or CA19-9 & $97.4 \%(38 / 39)$ & NA & NA & NA & NA \\
\hline ADAMTS1 + CA19-9 & $87.2 \%(34 / 39)$ & NA & NA & NA & NA \\
\hline$B N C 1+C A 19-9$ & $89.2 \%(33 / 37)$ & NA & NA & NA & NA \\
\hline
\end{tabular}

N/A: Not applicable 


\section{a ADAMTS1}

ROC: 0.9127

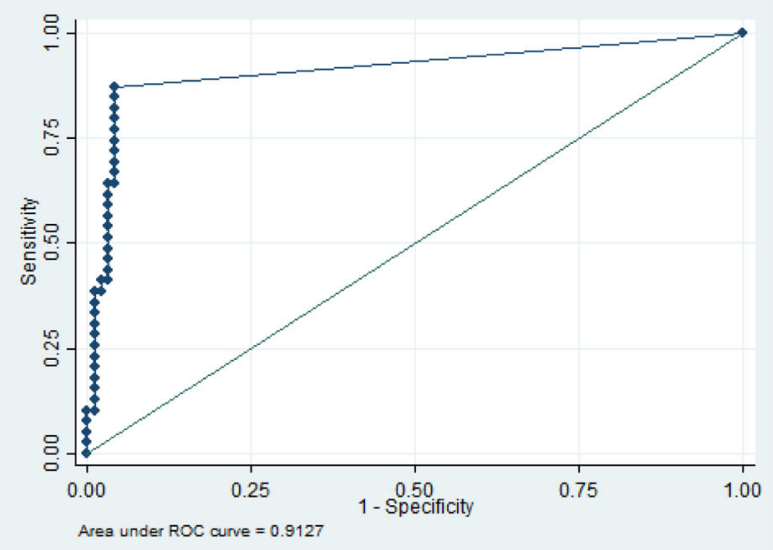

b BNC1

ROC: 0.7949

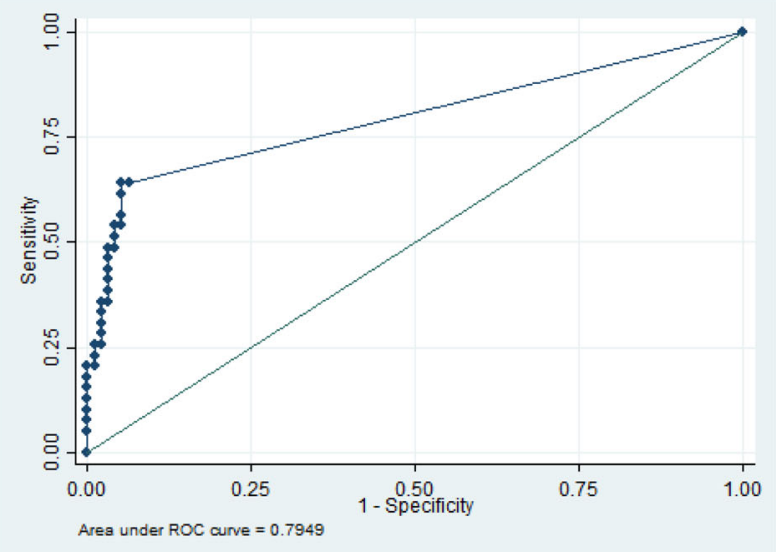

\section{c ADAMST1 + BNC1}

ROC: 0.9536

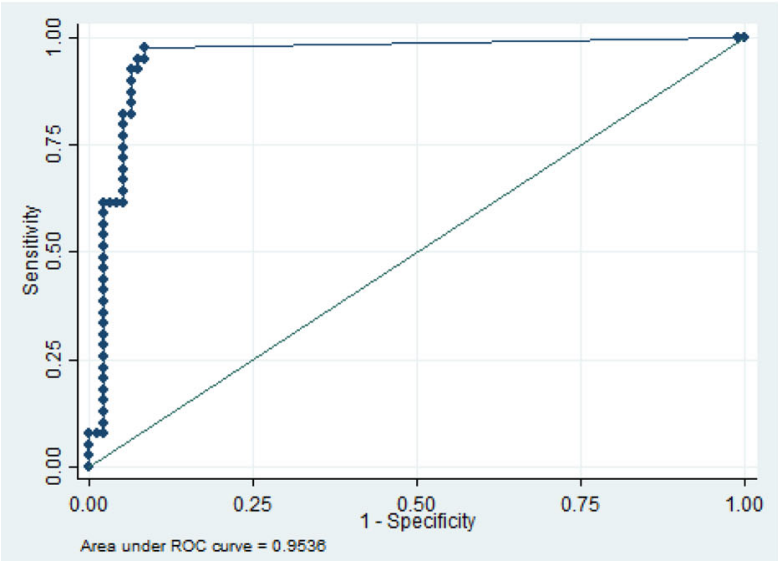

Fig. 2 Sensitivity and specificity of both genes. ROC curves for various genes. (a, b) ROC curves are represented for individual genes (ADAMTS1 and $B N C 1)$ and $\mathbf{c}$ combined methylation status of the genes (ADAMTS1 + BNC1) from the plasma samples 
CA 19-9 is currently the only biomarker routinely utilized clinically in the management of pancreatic cancer, generally as a method for surveillance of tumor recurrence. It can be used in the diagnostic workup of patients with suspicious lesions on imaging studies or patients with symptoms of pancreatic cancer such as obstructive jaundice. However, it is commonly negative in patients with pancreatic cancer and is not a reliable test for the detection of pancreatic cancer even in high-risk symptomatic patients, patients with pancreatitis, patients lacking Lewis antigens, and patients with early-stage cancer [19]. We showed this in our cohort, with stage I and stage II cancers demonstrating a positive CA19-9 in only $57.1 \%$ and $44.1 \%$, respectively (Table 3 ). We now show that a two-gene methylation panel (ADAMTS1 and/or $B N C 1$ ) demonstrated significant improvement in the detection rate, showing an even higher rate of positivity in stage I and stage II cancers $(100 \%$ and $94.4 \%$, respectively). However, the addition of CA 19-9 to the two-gene methylation panel did not show any further improvement.

We report a sensitivity and specificity of $87.2 \%$ and 95.8\% when considering the status of ADAMTS1, and $64.1 \%$ and $93.7 \%$ in the case of $B N C 1$. When considering the combination gene panel (ADAMTS1 and/or BNC1), the sensitivity and specificity increased to $97.4 \%$ and 91.6\% respectively. The high positivity $(87.5 \%)$ observed in patients with pancreatitis suggests that these markers would be most useful in cases in which chronic pancreatitis can be excluded. Validation investigations in the future should focus on identifying patient populations for whom this panel may not be as predictive, such as those with a history of pancreatitis.

In a previously published study, we had shown that considering both markers results in an overall sensitivity and specificity of $81 \%$ and $85 \%$ respectively. In this follow-up study, we have continued to optimize our panel, replacing SYBR green with a more specific TaqMan probe results in improvements in both sensitivity (97.4\%) and specificity (91.6\%). Also, testing our methylation panel on a larger control cohort was helpful in validating the sensitivity and specificity of the two-gene biomarker panel.

Due to its relatively low incidence rate compared to other types of cancer, population-based screening of pancreatic cancer is not reasonable or cost-effective. However, recent publications have shown benefits from screening specific high-risk populations [51-53]. Eight to $10 \%$ of patients with a family history of pancreatic cancer or a germline mutation would be candidates for screening along with patients who are long-term smokers [54-59]. Current diagnostic strategies include invasive techniques, offering endoscopic ultrasound screening of selected high-risk groups, or methods that involve extensive use of radiation-based screening [60].
The reduction in lung cancer mortality after using computed tomographic scan (CT scan) for the screening of in-risk population has already been shown by National Lung Screening Trial Research Team [61]. Non-invasive tests which use blood-based biomarkers, such as our suggested one, could be very helpful for the screening of asymptomatic patients, as it reduces the necessity of performing more invasive and expensive tests or biopsies.

Additionally, accurate blood-based biomarkers can be used for following up of previously diagnosed or suspicious pancreatic cancer cases $[62,63]$. A recently published study evaluated cancer cell-derived exosomes as a biomarker for pancreatic cancer with promising results [64]. However, this study did not describe the characteristics of the control group, which is essential in determining whether it is representative of the test group from other aspects besides the pancreatic cancer. Furthermore, the previous study included a large number of stage IV patients $(21.4 \%)$, but a small number of stage I patients $(2.6 \%)$, which is the stage at which intervention would be most beneficial and therefore should be the focus of a biomarker study [64, 65].

Our study now identifies a two-gene panel with highly promising sensitivity and specificity for detection of earliest stages of pancreatic cancer (sensitivity $97.4 \%$ and specificity 91.6\%) with an AUC 0.95 (CI 0.91-0.98) as compared to the sensitivity of $55.9 \%$ for CA $19-9$. The recent FDA approval of DNA methylation-based biomarkers in colorectal cancer highlights the feasibility of our approach as an economical mode of screening high-risk patients.

\section{Materials and methods Clinical samples}

The Institutional Review Boards of Johns Hopkins University approved the present study. A total of 142 samples were used for this study (Table 1). Patients with cancer undergoing surgical resection for infiltrating ductal adenocarcinoma of the pancreas were enrolled and blood samples were collected prior to incision $(n=39)$. A control group $(n=95)$ of volunteers was analyzed. The control cohorts enrolled in this study were age-matched patients attending an outpatient clinic for chronic diseases such as diabetes mellitus and hypertension. The exclusion criteria for controls included any history of malignancy and an age of less than 21 years old. In addition, we included eight patients who underwent pancreatic resection due to complicated and long-standing chronic pancreatitis (median years with disease prior to surgery, 84 months) (Table 1). All cohorts were consented and clinical information was obtained as per HIPAA regulations, including pre-operative CA19-9 level for cancer cases (in some cases, the data were not available and this is indicated in respective tables, and available total number were used for calculations). In addition, blood 
samples were collected and analyzed as per the institutional IRB protocol (NA00033085).

\section{DNA extraction and bisulfite conversion}

The blood specimens were collected using the standard operating procedures, processed using Ficoll-paque (GE health-care), frozen within $2 \mathrm{~h}$ of collection, and stored at $-80^{\circ} \mathrm{C}$ until utilized. Samples were collected before any surgery or chemotherapeutic treatment. All samples were collected with full institutional IRB approval from the involved centers and informed consent from all individuals who donated blood samples. Plasma obtained after centrifugation was aliquoted into multiple aliquot tubes and stored at $-80^{\circ} \mathrm{C}$. cfDNA was isolated from 2 $\mathrm{mL}$ of plasma using novel $\mathrm{MOB}$ method as described previously [66, 67]. In brief, MOB is a single-tube processing technique that employs silica-coated magnetic beads for both DNA extraction and bisulfite treatment that results in significant improvements in amplifiable bisulfite-converted DNA over conventional techniques $[28,29,66]$.

\section{Quantitative methylation-specific PCR}

We designed our QMSP experiments based on the minimum information for publication of quantitative real-time polymerase chain reaction (PCR) experiment (MIQE) guidelines [68]. Sensitive and specific TaqMan probe-based methods (IDT Inc.) were used for methylation analysis. Primer 3 and Meth Primer programs were used to design all our primers and probes (IDT Inc.). All primer sequences used in the evaluation are listed in Additional file 1: Table S2 and Table S3). Quantitative real-time methylation-specific PCR (QMSP) using StepOnePlus ${ }^{\text {Th }}$ Real-Time PCR System (Thermo Scientific) quantified bisulfite-converted cfDNA. The PCR mixture consisted of $2 \mu \mathrm{l}$ of bisulfite-converted DNA, $200 \mathrm{nM}$ of sense and anti-sense oligonucleotides, 100 $\mathrm{nM}$ probe, $100 \mathrm{nM}$ of fluorescein reference dye (Life Technologies), $1.67 \mathrm{mM}$ dNTPs (Thermo Scientific), and $1 \mu \mathrm{l}$ of Platinum $\mathrm{Taq}^{\circ}$ DNA Polymerase (Invitrogen). The master mix contained $16.6 \mathrm{mM}\left(\mathrm{NH}_{4}\right)_{2} \mathrm{SO}_{4}, 67 \mathrm{mM}$ Tris $\mathrm{pH}$ 8.8, 6.7 $\mathrm{mM} \mathrm{MgCl}$, and $10 \mathrm{mM} \beta$-mercaptoethanol in nuclease-free water. Amplification was performed on bisulfite-converted cfDNA with platinum Taq (Invitrogen) in a total volume of $25 \mu \mathrm{l}$ reaction. Amplification consisted of an initial activation for $10 \mathrm{~min}$ at $95^{\circ} \mathrm{C}$, followed by 45 cycles of melting at $95^{\circ} \mathrm{C}$ for $30 \mathrm{~s}$, annealing at $60^{\circ} \mathrm{C}$ for $30 \mathrm{~s}$, and extension at $72^{\circ} \mathrm{C}$ for $30 \mathrm{~s}$. All reactions were performed in triplicates using non-template or water samples as negative controls and CpG methylated Jurkat genomic DNA (IVD) as positive controls (Life Technologies). The quantitative methylation-specific assays were normalized to the levels of the housekeeper gene $\beta$-actin. All reactions were performed in triplicate and mean values were considered for statistical analyses.

\section{Statistical analysis}

Stata/MP 14.2 (StataCorp, College Station, Texas) was used for all statistical analysis. Wilcoxon rank-sum test was used to assess the statistical significance of methylation level differences between cancer and control groups. Data was analyzed using ROC analysis using $2^{-\Delta \mathrm{Ct}}$ values to determine the performance of individual markers which were used for combined detection analysis as described elsewhere [16]. The area under the curve obtained from a ROC curve analysis was used to test the biomarker accuracy. Sensitivity and specificity for each gene was calculated separately and in combination using the cutoff values obtained from the ROC curve.

\section{Additional file}

Additional file 1: Table S1. Surgical information. Table S2. Oligonucleotides probe sequences. Table S3. Oligonucleotides primer and probe design. (DOCX $36 \mathrm{~kb}$ )

\begin{abstract}
Abbreviations
ADAMTS1: A disintegrin and metalloproteinase with thrombospondin motifs; AUC: Area under the curve; BNC1: Zinc finger protein basonuclin-1; CfDNA: Cell-free DNA; CRC: Colorectal cancer; MOB: Methylation on beads; PCR: Polymerase chain reaction; QMSP: Quantitative real-time methylationspecific PCR; ROC: Receiver operating characteristic curve
\end{abstract}

\section{Acknowledgements}

We thank all our patients and control groups for participation in the study. In addition, we thank all Research Platform staff for assistance with sample collection and patient-care logistics.

\section{Funding}

Research reported in this publication was supported by National Institutes of Health under award number R01CA185357 and JHU T32 grant.

\section{Availability of data and materials}

All data is available from the corresponding author.

\section{Authors' contributions}

NA conceived and designed the study, obtained financial support, analyzed the data, and drafted the manuscript. ME and LL collected and processed the samples, optimized and performed the experiments, analyzed the data, and drafted the manuscript. EA optimized initial experiments. AS analyzed data and drafted the manuscript. NS analyzed the data and drafted the manuscript. JKC contributed to the data analysis and statistics. $\mathrm{NMH}, \mathrm{VY}, \mathrm{BH}$, ZK, FT, RK, MR, MT, and TRP read and approved the final manuscript. All authors provided samples, read, and approved the final manuscript.

\section{Ethics approval and consent to participate}

The Institutional Review Boards of Johns Hopkins University approved the present study. In addition, blood samples were collected and analyzed as per institutional IRB protocol (NA00033085).

\section{Consent for publication}

Not applicable

\section{Competing interests}

NA has received grant funding from Cepheid and Astex and has served as a consultant to Ethicon. NA has licensed methylation Biomarkers (BNC1 and ADAMTS1) to Cepheid Inc. All other authors declare that they have no competing interests. 


\section{Publisher's Note}

Springer Nature remains neutral with regard to jurisdictional claims in published maps and institutional affiliations.

\section{Author details \\ 'Department of Surgery, The Johns Hopkins University School of Medicine, Baltimore, MD, USA. ${ }^{2}$ Department of Oncology, The Sidney Kimmel Comprehensive Cancer Center, The Johns Hopkins University School of Medicine, Baltimore, MD, USA. ${ }^{3}$ Johns Hopkins Institute for NanoBioTechnology, The Johns Hopkins University, Baltimore, MD, USA. ${ }^{4}$ Department of Pathology, Memorial Sloan Kettering Cancer Center, New York, USA. ${ }^{5}$ Department of Pathology, The Johns Hopkins University School of Medicine, Baltimore, MD, USA. ${ }^{6}$ The Sol Goldman Pancreatic Cancer Research Center, The Sidney Kimmel Comprehensive Cancer Center, Johns Hopkins Hospital, Baltimore, MD, USA. Department of Surgery, Yale-New Haven Health, Yale University, School of Medicine, P.O. Box 208062, New Haven, CT 06520-8062, USA.}

Received: 7 August 2018 Accepted: 10 March 2019

\section{Published online: 05 April 2019}

\section{References}

1. Siegel RL, Miller KD, Jemal A. Cancer statistics, 2017. CA Cancer J Clin. 2017; 67(1):7-30.

2. Bosetti $C$, et al. Pancreatic cancer: overview of descriptive epidemiology. Mol Carcinog. 2012:51(1):3-13.

3. Worni $M$, et al. Modest improvement in overall survival for patients with metastatic pancreatic cancer: a trend analysis using the surveillance, epidemiology, and end results registry from 1988 to 2008. Pancreas. 2013; 42(7):1157-63.

4. Karmazanovsky G, et al. Pancreatic head cancer: accuracy of CT in determination of resectability. Abdom Imaging. 2005;30(4):488-500.

5. Garrido-Laguna I, Hidalgo M. Pancreatic cancer: from state-of-the-art treatments to promising novel therapies. Nat Rev Clin Oncol. 2015;12(6): 319-34

6. Cancer Stat Facts: Pancreatic Cancer. Pancreatic Cancer - Cancer Stat Facts 2018. https://seer.cancer.gov/statfacts/html/pancreas.html.

7. Ahuja N, Sharma AR, Baylin SB. Epigenetic therapeutics: a new weapon in the war against cancer. Annu Rev Med. 2016;67:73-89.

8. Esteller $\mathrm{M}$, et al. Detection of aberrant promoter hypermethylation of tumor suppressor genes in serum DNA from non-small cell lung cancer patients. Cancer Res. 1999;59(1):67-70.

9. Chan TA, Baylin SB. Epigenetic biomarkers. Curr Top Microbiol Immunol. 2012:355:189-216.

10. Hong $\mathrm{SM}_{\text {, et }}$ al. Multiple genes are hypermethylated in intraductal papillary mucinous neoplasms of the pancreas. Mod Pathol. 2008:21(12):1499-507.

11. Hong SM, et al. Genome-wide CpG island profiling of intraductal papillary mucinous neoplasms of the pancreas. Clin Cancer Res. 2012:18(3):700-12.

12. Sato $\mathrm{N}$, et al. $\mathrm{CpG}$ island methylation profile of pancreatic intraepithelial neoplasia. Mod Pathol. 2008:21(3):238-44.

13. Schuebel KE, et al. Comparing the DNA hypermethylome with gene mutations in human colorectal cancer. PLoS Genet. 2007;3(9):1709-23.

14. Jeschke J, et al. Biomarkers for detection and prognosis of breast cancer identified by a functional hypermethylome screen. Epigenetics. 2012;7(7):701-9.

15. Yi JM, et al. Novel methylation biomarker panel for the early detection of pancreatic cancer. Clin Cancer Res. 2013;19(23):6544-55.

16. Hulbert A, et al. Early detection of lung cancer using DNA promoter hypermethylation in plasma and sputum. Clin Cancer Res. 2017;23(8):1998-2005.

17. Matsubayashi $\mathrm{H}$, et al. DNA methylation alterations in the pancreatic juice of patients with suspected pancreatic disease. Cancer Res. 2006;66(2):1208-17.

18. Kanda M, et al. Mutant GNAS detected in duodenal collections of secretinstimulated pancreatic juice indicates the presence or emergence of pancreatic cysts. Gut. 2013;62(7):1024-33.

19. Frebourg T, et al. The evaluation of CA 19-9 antigen level in the early detection of pancreatic cancer. A prospective study of 866 patients. Cancer. 1988:62(11):2287-90.

20. Goonetilleke KS, et al. Diagnostic and prognostic value of plasma tumor M2 pyruvate kinase in periampullary cancer: evidence for a novel biological marker of adverse prognosis. Pancreas. 2007;34(3):318-24.

21. Kaur $\mathrm{S}$, et al. Early diagnosis of pancreatic cancer: challenges and new developments. Biomark Med. 2012;6(5):597-612.
22. Steinberg $W$. The clinical utility of the CA 19-9 tumor-associated antigen. Am J Gastroenterol. 1990;85(4):350-5.

23. Leon SA, et al. Free DNA in the serum of cancer patients and the effect of therapy. Cancer Res. 1977:37(3):646-50.

24. Gahan PB, Swaminathan R. Circulating nucleic acids in plasma and serum. Recent developments. Ann N Y Acad Sci. 2008;1137:1-6.

25. Karampini E, McCaughan F. Circulating DNA in solid organ cancers-analysis and clinical application. QJM. Int J Med. 2016;109(4):223-7.

26. Cohen JD, et al. Combined circulating tumor DNA and protein biomarkerbased liquid biopsy for the earlier detection of pancreatic cancers. Proc Natl Acad Sci U S A. 2017;114(38):10202-7.

27. Cohen JD, et al. Detection and localization of surgically resectable cancers with a multi-analyte blood test. Science. 2018;359(6378):926-30.

28. Guzzetta AA, et al. The promise of methylation on beads for cancer detection and treatment. Expert Rev Mol Diagn. 2014;14(7):845-52.

29. Keeley $B$, et al. Extraction and processing of circulating DNA from large sample volumes using methylation on beads for the detection of rare epigenetic events. Clin Chim Acta. 2013;425:169-75.

30. Pandol S, et al. Epidemiology, risk factors, and the promotion of pancreatic cancer: role of the stellate cell. J Gastroenterol Hepatol. 2012;27(Suppl 2): 127-34.

31. Yeo TP. Demographics, epidemiology, and inheritance of pancreatic ductal adenocarcinoma. Semin Oncol. 2015:42(1):8-18.

32. Midha S, Chawla S, Garg PK. Modifiable and non-modifiable risk factors for pancreatic cancer: a review. Cancer Lett. 2016:381(1):269-77.

33. A stool DNA test (Cologuard) for colorectal cancer screening. Jama. 2014; 312(23):2566.

34. Cai L, Hood S, Kallam E, Overman D, Barker K, et al. Epi proColon ${ }^{\oplus}$ : Use of a non-invasive SEPT9 gene methylation blood test for colorectal cancer screening: A national laboratory experience. J Clin Epigenet. 2018;4:7. https://doi.org/10.21767/2472-1158.100092.

35. de Vos T, et al. Circulating methylated SEPT9 DNA in plasma is a biomarker for colorectal cancer. Clin Chem. 2009;55(7):1337-46.

36. Warren JD, et al. Septin 9 methylated DNA is a sensitive and specific blood test for colorectal cancer. BMC Med. 2011;9:133.

37. Rakovitch $\mathrm{E}$, et al. Multigene expression assay and benefit of radiotherapy after breast conservation in ductal carcinoma in situ. J Natl Cancer Inst. 2017:109(4):djw256.

38. Renfro LA, et al. Prospective evaluation of a 12-gene assay on patient treatment decisions and physician confidence in mismatch repair proficient stage IIA colon cancer. Clin Colorectal Cancer. 2017;16(1):23-30.

39. Van Den Eeden SK, et al. A biopsy-based 17-gene genomic prostate score as a predictor of metastases and prostate cancer death in surgically treated men with clinically localized disease. Eur Urol. 2018; 73(1):129-38.

40. Esteller M, et al. A gene hypermethylation profile of human cancer. Cancer Res. 2001:61(8):3225-9.

41. Tsai HC, Baylin SB. Cancer epigenetics: linking basic biology to clinical medicine. Cell Res. 2011:21(3):502-17.

42. Howlader N, Noone AM, Krapcho M, Miller D, Bishop K, Kosary CL, Yu M Ruhl J, Tatalovich Z, Mariotto A, Lewis DR, Chen HS, Feuer EJ, Cronin KA. SEER Cancer Statistics Review, 1975-2014. Bethesda: National Cancer Institute; 2017.

43. Henschke $\mathrm{Cl}$, et al. Survival of patients with stage I lung cancer detected on CT screening. N Engl J Med. 2006:355(17):1763-71.

44. American Cancer Society. Colorectal Cancer Facts \& Figures 2017-2019. Atlanta: American Cancer Society; 2017. p. 2017.

45. Egawa S, et al. Clinicopathological aspects of small pancreatic cancer. Pancreas. 2004;28(3):235-40.

46. Hur C, et al. Early pancreatic ductal adenocarcinoma survival is dependent on size: positive implications for future targeted screening. Pancreas. 2016 45(7):1062-6.

47. Marchegiani $\mathrm{G}$, et al. Does size matter in pancreatic cancer?: reappraisal of tumour dimension as a predictor of outcome beyond the TNM. Ann Surg. 2017;266(1):142-8

48. Lennon AM, et al. The early detection of pancreatic cancer: what will it take to diagnose and treat curable pancreatic neoplasia? Cancer Res. 2014:74(13):3381-9.

49. Wagner $\mathrm{M}$, et al. Curative resection is the single most important factor determining outcome in patients with pancreatic adenocarcinoma. $\mathrm{Br} \mathrm{J}$ Surg. 2004;91(5):586-94. 
50. Griffin JF, Poruk KE, Wolfgang CL. Pancreatic cancer surgery: past, present, and future. Chin J Cancer Res. 2015;27(4):332-48.

51. Rulyak SJ, et al. Cost-effectiveness of pancreatic cancer screening in familial pancreatic cancer kindreds. Gastrointest Endosc. 2003;57(1):23-9.

52. Shin EJ, Canto MI. Pancreatic cancer screening. Gastroenterol Clin N Am. 2012:41(1):143-57.

53. Verna EC, et al. Pancreatic cancer screening in a prospective cohort of highrisk patients: a comprehensive strategy of imaging and genetics. Clin Cancer Res. 2010;16(20):5028-37.

54. Li D, et al. Pancreatic cancer. Lancet. 2004;363(9414):1049-57.

55. Petersen GM, et al. Pancreatic cancer genetic epidemiology consortium. Cancer Epidemiol Biomarkers Prev. 2006;15(4):704-10.

56. Rustgi AK. Familial pancreatic cancer: genetic advances. Genes Dev. 2014; 28(1):1-7.

57. Yeo TP, et al. Pancreatic cancer. Curr Probl Cancer. 2002;26(4):176-275.

58. Humphris $\mathrm{JL}$, et al. Clinical and pathologic features of familial pancreatic cancer. Cancer. 2014;120(23):3669-75.

59. Petersen GM. Familial pancreatic adenocarcinoma. Hematol Oncol Clin North Am. 2015;29(4):641-53.

60. Canto Ml, et al. International Cancer of the Pancreas Screening (CAPS) Consortium summit on the management of patients with increased risk for familial pancreatic cancer. Gut. 2013;62(3):339-47.

61. Aberle DR, et al. Reduced lung-cancer mortality with low-dose computed tomographic screening. N Engl J Med. 2011;365(5):395-409.

62. Anderson MA, et al. Alcohol and tobacco lower the age of presentation in sporadic pancreatic cancer in a dose-dependent manner: a multicenter study. Am J Gastroenterol. 2012;107(11):1730-9.

63. Pang $\mathrm{T}$, et al. A novel scoring system to analyze combined effect of lifestyle factors on pancreatic cancer risk: a retrospective case-control study. Sci Rep. 2017:7(1):13657.

64. Melo SA, et al. Glypican-1 identifies cancer exosomes and detects early pancreatic cancer. Nature. 2015;523(7559):177-82.

65. Babic A, Wolpin BM. Circulating exosomes in pancreatic cancer: will they succeed on the long, littered road to early detection marker? Clin Chem. 2016;62(2):307-9.

66. Bailey VJ, et al. Single-tube analysis of DNA methylation with silica superparamagnetic beads. Clin Chem. 2010:56(6):1022-5.

67. Bailey VJ, et al. MS-qFRET: a quantum dot-based method for analysis of DNA methylation. Genome Res. 2009;19(8):1455-61.

68. Bustin SA, et al. The MIQE guidelines: minimum information for publication of quantitative real-time PCR experiments. Clin Chem. 2009;55(4):611-22.

Ready to submit your research? Choose BMC and benefit from:

- fast, convenient online submission

- thorough peer review by experienced researchers in your field

- rapid publication on acceptance

- support for research data, including large and complex data types

- gold Open Access which fosters wider collaboration and increased citations

- maximum visibility for your research: over $100 \mathrm{M}$ website views per year

At $\mathrm{BMC}$, research is always in progress.

Learn more biomedcentral.com/submissions 